\title{
Características de los donantes voluntarios de sangre en universidades de Neiva, Huila
}

\author{
Characteristics of voluntary blood donors at universities in Neiva, Huila \\ Características de doadores de sangue voluntários em universidades de Neiva, Huila
}

\author{
José Vladimir Guzmán-Rivera ${ }^{1}$ \\ Diana Cristina Alvira-Guauña ${ }^{2}$ \\ Marly Hasbleidy Morales-Pérez ${ }^{3}$
}

\section{Resumen}

Objetivo: Describir las características, frecuencia y distribución de los donantes voluntarios de sangre en las Universidades de Neiva Huila, durante el período 20132017. Materiales y métodos: Estudio observacional descriptivo de corte transversal para los periodos de enero 2013 a diciembre 2017, basado en fuentes secundarias suministradas por la Red Nacional de Bancos de Sangre a través del Banco de Sangre de Neiva. Se realizó un muestreo de donantes por conveniencia que cumplieran con criterios de inclusión y exclusión definidos para el estudio. Las variables que se analizaron fueron grupos de edad, género, grupo sanguíneo $\mathrm{ABO}$ y factor $\mathrm{Rh}$. Resultados: Durante el periodo de estudio se realizaron 6.547 donaciones de sangre voluntarias, de las cuales el $85,5 \%$ (I.C ${ }_{95 \%}: 84,6-86,3$ ) fueron ocasionales y el $14,5 \%$ (I.C ${ }_{95 \%}: 13,6-15,6$ ) habituales; el género masculino generó la mayor parte de las donaciones voluntarias habituales en el 68\% (I.C $\left.{ }_{95 \%}: 64,9-70,9\right)$, se identificó que el grupo poblacional en edades de 19-29 años, para ambos géneros, aporto el 87,5\% (I.C ${ }_{95 \%}$ : 85,2-89,4); el grupo sanguíneo que prevaleció en los donantes voluntarios fue el grupo O en el 61,6\% (I.C $95 \%$ : 57,2-63,4) y el factor Rh positivo en el 98,5\% de la población donante. Conclusión: Se presentó una disminución cercana al $90 \%$ en los donantes habituales posterior a tercera década de la vida, los hombres tuvieron una mayor participación en las jornadas de promoción y captación de sangre durante el periodo de estudio, dado a que los hombres pueden realizar un mayor número de donaciones en el año en comparación con las mujeres.

Palabras Clave: Donantes de sangre; Sangre; Voluntarios; Bancos de Sangre; Sistema del grupo sanguíneo ABO.

\section{Resumo}

Objetivo: Descrever as características, frequência e distribuição de doadores voluntários de sangue nas Universidades de Neiva Huila, durante o período de 2013 a 2017. Materiais e Métodos: Estudo descritivo transversal observacional entre os períodos

\begin{abstract}
Autor de correspondencia"
1* Enfermero. Especialista en Salud Ocupacional. Magíster en Epidemiologia. Docente, Programa de Enfermería, Integrante grupo de Investigación Salud y Grupos Vulnerables, Universidad Surcolombiana. Enfermero E.S.E Hospital Universitario Hernando Moncaleano Perdomo. Neiva, Colombia. Correo: jvguzmanr@hotmail. com (iD)

${ }^{2}$ Estudiante de Enfermería. Programa de Enfermería, Integrante grupo de Investigación Salud y Grupos Vulnerables, Universidad Surcolombiana. Neiva, Colombia. Correo: dianacra99@gmail.com ic

${ }^{3}$ Estudiante de Enfermería. Programa de Enfermería, Integrante grupo de Investigación Salud y Grupos Vulnerables Universidad Surcolombiana. Neiva, Colombia. Correo:xtartis@gmail.com ic
\end{abstract}

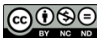

(c) Universidad Francisco de Paula Santander. Este es un artículo bajo la licencia CC-BY-NC-ND

Recibido: 6 abril 2020. Aprobado: 6 julio 2020.

Para citar este artículo/ To reference this article/ Para citar este artigo/

Guzmán-Rivera José V, Alvira-Guauña DC, Morales-Pérez MH. Características de los donantes voluntarios de sangre en universidades de Neiva, Huila. Rev. cienc. cuidad. 2020; 17(3):75-85. https://doi.org/10.22463/17949831.2383 
de janeiro de 2013 a dezembro de 2017 com base em fontes secundárias fornecidas pela Rede Nacional de Bancos de Sangue através do Banco de Sangue do Neiva; Foi realizada uma amostragem de doadores por conveniência, atendendo aos critérios de inclusão e exclusão definidos para o estudo. As variáveis analisadas foram: faixa etária, gênero, grupo sanguíneo ABO e fator Rh. Resultados: Durante o período de estudo, foram feitas 6.547 doações voluntárias de sangue, das quais 85,5\% (95\%: $84,6-86,3$ ) foram feitas com base nos seguintes $14,5 \%$ (I.C 95\%: 13,6-15,6) por regular. O gênero masculino gerou a maior parte das doações voluntárias habituais em 68\% (I.C 95\%: 64,9-70,9), o grupo populacional de 19 a 29 anos foi identificado para ambos os sexos, fornecendo 87,5\% (I.C 95\%: 85,2-89,4); o grupo sanguíneo que mais prevaleceu nos doadores voluntários foi o grupo O em 61,6\% (I.C 95\%: 57,2-63,4) e o fator Rh positivo em $98,5 \%$ da população doadora. Conclusão: Houve uma redução de quase $90 \%$ nos doadores regulares após a terceira década de vida, Os homens tiveram maior participação nos dias de promoção e coleta de sangue durante o período de estudo, uma vez que os homens podem fazer um maior número de doações no ano em comparação com as mulheres.

Palavras chave: Doadores de Sangue; Sangue; Voluntários; Bancos de Sangue; Sistema ABO de Grupos Sanguíneos.

\section{Abstract}

Objective: To describe the characteristics of voluntary blood donors in the Universities of Neiva Huila, during the period 2013-2017. Materials and methods: Cross-sectional descriptive observational study, for the periods from January 2013 to December 2017, based on secondary sources provided by the National Network of Blood Banks. A convenience sampling was performed of the donors registered in the database, who met the inclusion and exclusion criteria defined for the study. The variables analyzed were age groups, gender, ABO blood group and Rh factor. Results: During the study period, 6,547 voluntary blood donations were made, of which $85.5 \%$ (95\% CI: 84.6-86.3) were occasional and $14.5 \%$ (95\% CI: 13 . 6-15.6) usual. The male gender generated most of the usual voluntary donations in $68 \%$ (95\% CI: 64.970.9), it was identified that the population group aged 19-29 years for both genders contributed $87,5 \%$ (95\% CI: 85.2-89.4); the most prevalent blood group in volunteer donors was the blood group O Rh factor Positive in 61.6\% (95\% CI: 57.2-63.4) and the $\mathrm{Rh}$ factor positive in $98.5 \%$ of the patients. donor population. Conclusion: There was a decrease close to $90 \%$ in habitual donors after the third decade of life. Men had a greater participation in the days of promotion and collection of blood during the study period, given that men can make a greater number of donations in the year compared to women.

Keywords: Population characteristics; Blood donors; ABO blood group system; Blood, Rh-Hr blood group system

\section{Introducción}

La sangre es un fluido corporal de gran importancia para mantener la vida de las personas, posee un volumen cercano a los 5 o 6 litros y representa cerca del 8\% del peso corporal de un adulto(1). En la actualidad, pese a los avances en salud este fluido no se ha logrado reemplazar sintéticamente debido a la complejidad de sus características y a su composición de eritrocitos, plaquetas, leucocitos y plasma; siendo este último una mezcla de proteínas, hormonas, iones, entre otras sustancias(1).La importancia de este fluido se puede evidenciar cuando una persona sufre de una pérdida significativa de sangre y necesita una transfusión de urgencia para preservar su vida. 
La transfusión sanguínea es un procedimiento médico que consiste en transferir sangre en la cantidad necesaria a la persona que lo requiera para normalizar su volemia (volumen total de sangre que circula dentro de un humano), por lo cual la recolección de sangre y captación de donantes sigue siendo un gran desafío debido a la alta demanda del fluido y la poca capacidad de abastecimiento, especialmente en países de bajos y medianos ingresos(2). Según datos recientes del Ministerio de Salud en Colombia(3), se recolectaron 830.000 unidades de sangre provenientes de donantes hombres en un 52,6\% y de mujeres en un 47,4\%. Al igual que en otros países como Brasil, la mayoría de los donantes por primera vez no regresan y solo el $22,1 \%$ se vuelven habituales (personas que donan al menos dos veces al año), mientras que un $77,9 \%$ tienden a ser nuevos donantes (personas que donan por primera vez)(4).

En Colombia existe una clasificación de los tipos de donantes de sangre, según su frecuencia y motivación, emitida en el 2018 por el Instituto Nacional de Salud y el Ministerio de Salud en el documento "Lineamiento técnico para la selección de donantes de sangre", en el cual se clasifican en: Donantes autólogos, aquellos que donan su sangre para ser reservada antes de un procedimiento quirúrgico; donantes voluntarios y altruistas quienes lo hacen para beneficiar a personas que en su mayoría son desconocidas del donante, sin recibir nada a cambio y sin un fin específico; donante por reposición que es aquel que dona su sangre para beneficiar a un familiar, amigo o paciente determinado que la requiere, "previo a un proceso de sensibilización y educación"; por último, los donantes dirigidos los cuales donan sangre para un receptor determinado(5).

Para garantizar la calidad de la sangre normalmente cada uno de los donantes debe diligenciar un instrumento de datos básicos, el cual sirve como prueba de selección previa a la donación; posteriormente, en el banco de sangre se realizan una serie de pruebas de laboratorio a las unidades donadas como lo menciona la Organización Mundial de la Salud (OMS)(6), para corroborar de forma precisa y confiable que la sangre esté libre de patógenos que puedan ocasionar efectos negativos en la persona a quien se le vaya a realizar la transfusión.

En la región de las Américas la Organización Panamericana de la Salud (OPS), cuenta con un plan regional para el mejoramiento de la disponibilidad de sangre y la seguridad de las transfusiones, un tema de importancia en la salud pública, que contribuye al logro de los objetivos 4,5 y 6 de desarrollo del milenio. Para lograrlos se implementó un enfoque multidisciplinario y coordinado para promoción de las donaciones de sangre y así poder garantizar suministros de sangre suficientes y seguros(7).

De acuerdo al artículo 28 del decreto 1571 de 1993(8), donar sangre es una acción solidaria sin remuneración; la recolección de este fluido se ha de realizar a través de distintos bancos de sangre habilitados y ubicados en diferentes regiones del país. Según el informe dado por Instituto Nacional de Salud(9), en la actualidad se cuenta con 81 de estos bancos registrados con un código único nacional, de los cuales 30 son públicos, 43 privados, 6 de la Cruz Roja y 2 de las fuerzas militares y de la policía. El departamento del Huila cuenta con tres bancos de sangre de carácter público ubicados en Neiva, Garzón y Pitalito. En la capital del departamento se encuentra uno de los bancos de sangre más importantes del país, adscrito a una reconocida Empresa Social del Estado de la región por ofertar servicios de salud de tercer y cuarto nivel de atención en salud. La Universidad Surcolombiana a través del convenio Docencia Servicio, desarrolla un proyecto de proyección social denominado "Promoción de la donación voluntaria y habitual de sangre en la comunidad huilense", liderado por docentes, estudiantes y egresados del programa de enfermería, en el que se fortalecen las campañas de promoción y captación voluntaria de sangre realizadas en la unidad móvil del banco de sangre, en pro de incentivar en la población huilense una cultura de donación.

Enmarcada en lo anterior, la presente investigación buscó describir las características, frecuencia y distribución de los donantes voluntarios de sangre en las diferentes universidades del municipio de Neiva, durante las jornadas de promoción y captación de sangre realizadas entre el periodo de 2013 al 2017. Este estudio permitirá el fortalecimiento de las estrategias de promoción en la donación voluntaria y habitual de sangre, teniendo en cuenta las características de la población objeto.

\section{Objetivos}

\section{Objetivo General}

Describir las características, frecuencia y distribución de los donantes voluntarios de sangre en las Universidades de Neiva Huila, durante el período 2013-2017. 


\section{Objetivos específicos}

ISSN-PRINT

1794-9831

E-ISSN 2322-7028

Vol. 17 No. 3

Sep - Dic 2020

Cúcuta, Colombia
- Establecer la frecuencia y distribución de las donaciones ocasionales y habituales de las universidades por año de donación.

-Identificar las características sociodemográficas de la población donante de sangre ocasional y habitual de las universidades por año.

- Determinar los grupos sanguíneos y factor $\mathrm{Rh}$ prevalentes en la población donante de sangre ocasional y habitual en universidades por año.

\section{Materiales y Métodos}

Estudio observacional, descriptivo de corte transversal, para los periodos de enero 2013 a diciembre 2017, basado en fuentes secundarias, anonimizadas y suministradas por la Red Nacional de Bancos de Sangre a través del Banco de Sangre de la ciudad de Neiva. Se realizó revisión de la base de datos del banco de sangre, la cual tiene la información consolidada a partir del instrumento auto administrado por los donantes, bajo la supervisión del personal de salud en el momento de la valoración, en la instalaciones de la unidad móvil durante las jornadas de promoción y captación de sangre realizadas en las universidades.

La población la conformaron 7421 donantes voluntarios de sangre en las nueve universidades del municipio, en la que podían donar: Docentes, administrativos y estudiantes, siempre y cuando cumplieran con los requisitos universales para la donación. La muestra se conformó con 6547 donantes voluntarios de sangre ocasionales y habituales. La selección de los participantes del estudio se realizó mediante un muestreo no probabilístico por conveniencia, en el que se definieron criterios de inclusión e exclusión, los cuales se mencionan a continuación:

Criterios de inclusión: donantes ocasionales quienes donaron una vez al año y donantes de sangre habitual aquellos que donaron sangre entre dos y cuatro veces al año durante el periodo de estudio.

Criterios de exclusión: donantes voluntarios por reposición, donantes por aféresis y donantes que tuvieran un sitio de donación diferente al definido en el estudio.
Con los datos obtenidos se consolida la información correspondiente a número de donaciones por año y características sociodemográficas como edad y género, el grupo sanguíneo ABO y el factor Rh. A partir de la cantidad de donaciones realizadas por el donante por año, se clasificaron en: Ocasionales: Una donación en determinado año; Habitual: dos a cuatro donaciones en determinado año. Para la organización de datos se elaboró una matriz, empleando el software Excel 2016, en donde se asignó un código numérico consecutivo a cada una de las variables del estudio. Para el análisis de los datos se utilizó el programa de análisis estadístico STATA versión 15 Licenciado, presentando los datos en frecuencias absolutas y porcentajes con sus respectivos intervalos de confianza (95\%).

De acuerdo con la resolución 8430 de 1993 del Ministerio de Salud, la presente investigación fue clasificada sin riesgo por emplear técnicas y métodos de investigación documental retrospectivos, sin que se realizaran intervenciones o modificación intencionada de las variables biológicas, fisiológicas, psicológicas o sociales de los individuos que participan en el estudio. La investigación fue avalada por el Comité de ética, bioética e investigación de la E.S.E Hospital Universitario Hernando Moncaleano de Neiva, mediante el acta Numero 007-002 del 17 de julio del 2018.

\section{Resultados}

Durante el periodo de estudio se presentaron 6.547 donaciones de sangre voluntarias en las universidades de Neiva (Huila), de las cuales el $85,5 \%$ (I.C $\left.{ }_{95 \%}: 84,6-86,3\right)$ de las donaciones provenían de donantes voluntarios ocasionales; se observó un incremento del $4.9 \%$ al comparar los registros del año 2017 frente al 2013 (Ver tabla 1).

Mientras que los donantes de sangre habitual representaron el 14,5\% (I.C ${ }_{95 \%}: 13,6-15,6$ ) del total de las donaciones de sangre voluntarias realizadas para el mismo periodo con un incremento del $0.6 \%$ en este tipo de donantes al comparar los registros del año 2017 frente al 2013 (Tabla 1). 
Tabla 1. Clasificación de los donantes voluntarios de sangre en las universidades de Neiva, 2013-2017.

\begin{tabular}{ccccccc}
\hline \multirow{2}{*}{ AÑ } & \multicolumn{2}{c}{ OCASIONALES } & \multicolumn{5}{c}{ HABITUALES } \\
& No & \% & IC 95\% & No & \% & IC 95\% \\
\hline 2013 & 880 & 13,4 & $12,6-14,2$ & 162 & 2,5 & $2,1-2,8$ \\
2014 & 1010 & 15,4 & $14,5-16,3$ & 165 & 2,5 & $2,1-2,9$ \\
2015 & 1153 & 17,6 & $16,7-18,5$ & 186 & 2,8 & $2,4-3,2$ \\
2016 & 1359 & 20,8 & $19,7-21,7$ & 228 & 3,5 & $3,0-3,9$ \\
2017 & 1198 & 18,3 & $17,3-19,2$ & 206 & 3,1 & $2,7-3,6$ \\
TOTAL & 5600 & 85,5 & $84,6-86,3$ & 947 & 14,5 & $13,6-15,3$ \\
\hline
\end{tabular}

ISSN-PRINT

$1794-9831$

E-ISSN 2322-7028

Vol. 17 No. 3

Sep - Dic 2020

Cúcuta, Colombia

Fuente: Elaboración propia a partir de la base de datos del banco de sangre.

De acuerdo con la información recolectada $\mathrm{y}$ procesada, se identificaron características como género y edad, donde se evidenció que el género masculino aportó el 68\% (I.C ${ }_{95 \%}: 64,9-70,9$ ) de las donaciones voluntarias de sangre habitual para el periodo de estudio, con un incremento del
2,5\% al comparar los registros del año 2017 frente al 2013. A pesar de que las mujeres no tuvieron un mayor porcentaje de participación en las jornadas de donación durante el periodo de estudio, se registró un incremento del 2,2\% al comparar el 2017 con el año 2013 (Ver tabla 2).

Tabla 2. Donantes voluntarios de sangre habitual por género, en las Universidades de Neiva Huila, 2013-2017.

\begin{tabular}{ccccccc}
\hline \multirow{2}{*}{ AÑO } & \multicolumn{3}{c}{ FEMENINO } & \multicolumn{3}{c}{ MASCULINO } \\
& Frecuencia & $\mathbf{\%}$ & IC 95\% & Frecuencia & \% & IC 95\% \\
\hline $\mathbf{2 0 1 3}$ & 50 & 5,3 & $4,0-6,8$ & 112 & 11,8 & $9,9.14,0$ \\
$\mathbf{2 0 1 4}$ & 47 & 5 & $3,7-6,5$ & 118 & 12,5 & $10,5-14,7$ \\
$\mathbf{2 0 1 5}$ & 54 & 5,7 & $4,4-7,3$ & 132 & 13,9 & $11,8-16,2$ \\
$\mathbf{2 0 1 6}$ & 81 & 8,6 & $6,9-10,5$ & 147 & 15,5 & $13,6-17,9$ \\
$\mathbf{2 0 1 7}$ & 71 & 7,5 & $5,9-9,3$ & 135 & 14,3 & $12,1-16,6$ \\
Total & 303 & 32 & $29,1-35,0$ & 644 & 68 & $64,9-70,9$ \\
\hline
\end{tabular}

Fuente: Elaboración propia a partir de la base de datos del banco de sangre.

Los adultos jóvenes, en rangos de edades entre los 20 y 29 años, fueron los principales donantes de sangre habitual en ambos géneros representando el 63\% (I.C $\left.{ }_{95 \%}: 59,9-66,0\right)$ del total de las donaciones habituales realizadas durante las jornadas de promoción y captación de sangre durante el periodo 2013-2017; además se evidenció un aumento del 3,9\% al comparar los años
2017 frente al 2013. Aunque la participación de los donantes habituales en edades de 18 y 19 años ha representado el 24,5\% (I.C ${ }_{95 \%}: 21,8$ $27,3)$ del total de las donaciones habituales, en este grupo poblacional se observó una reducción del $0,5 \%$ para el último año en comparación con el año de inicio del periodo analizado (Tabla 3 ). 
Tabla 3. Donantes voluntarios de sangre habitual por rangos de edad, en ambos géneros, Neiva Huila $2013-2017$.

ISSN-PRINT

1794-9831

E-ISSN 2322-7028

Vol. 17 No. 3

Sep - Dic 2020

Cúcuta, Colombia

\begin{tabular}{|c|c|c|c|c|c|c|c|c|c|c|c|c|c|c|c|c|c|c|}
\hline \multirow{2}{*}{ AÑO } & \multicolumn{3}{|c|}{$18-19$} & \multicolumn{3}{|c|}{$20-29$} & \multicolumn{3}{|c|}{ 30-39 } & \multicolumn{3}{|c|}{ 40-49 } & \multicolumn{3}{|c|}{$50-59$} & \multicolumn{3}{|c|}{$60-69$} \\
\hline & No & $\%$ & IC 95\% & No & $\%$ & IC 95\% & No & $\%$ & IC 95\% & No & $\%$ & IC $95 \%$ & No & $\%$ & IC 95\% & No & $\%$ & IC $95 \%$ \\
\hline 2013 & 46 & 4,9 & $3,6-6,4$ & 99 & 61,1 & $53,4-68,3$ & 7 & 4,3 & $2,1-8,6$ & 3 & 1,90 & $0,6-5,3$ & 5 & 3,10 & $1,3-4,4$ & 2 & 1,20 & $0,3-44$ \\
\hline 2014 & 50 & 5,3 & $4,0-6,8$ & 96 & 58,2 & $50,5-65,4$ & 7 & 4,2 & $2,1-8,5$ & 4 & 2,40 & $0,9-6,1$ & 7 & 4,20 & $2,1-8,5$ & 1 & 0,60 & $0,1-3,4$ \\
\hline 2015 & 50 & 5,3 & $4,0-6,8$ & 106 & 57,0 & $49,8-63,9$ & 16 & 8,6 & $5,4-13,5$ & 7 & 3,80 & $1,8-7,6$ & 7 & 3,80 & $1,8-7,6$ & 0 & 0,00 & $0,0-2,0$ \\
\hline 2016 & 44 & 4,6 & $3,4-6,1$ & 160 & 70,2 & $63,9-75,7$ & 16 & 7,0 & $4,4-11,1$ & 5 & 2,20 & $0,9-5,0$ & 3 & 1,30 & $0,4-3,8$ & 0 & 0,00 & $0,0-2,0$ \\
\hline 2017 & 42 & 4,4 & $3,3-5,9$ & 136 & 66,0 & $59,3-72,1$ & 13 & 6,3 & $3,7-10,5$ & 7 & 3,40 & $1,7-6,8$ & 8 & 3,90 & $2,0-7,5$ & 0 & 0,00 & $0,0-2,0$ \\
\hline TOTAL & 232 & 24,5 & $21,8-27,3$ & 597 & 63,0 & $59,9-66,0$ & 59 & 6,2 & $4,8-7,9$ & 26 & 2,75 & $1,8-3,9$ & 30 & 3,17 & $2,2-4,4$ & 3 & 0,32 & $0,1-0,9$ \\
\hline
\end{tabular}

Fuente: Elaboración propia a partir de la base de datos del banco de sangre.

Entre los donantes voluntarios de sangre habitual, grupo que predomino entre la población donante se encontró que en el 92,4\% (I.C $95 \%$ : 90,5-93,9) habitual con el 60,4\% (I.C $\left.{ }_{95 \%}: 57,2-63,4\right)$, seguitenían el factor Rh positivo; siendo el grupo $\mathrm{O}$, el do por el grupo $\mathrm{A}$, respectivamente (ver tabla 4).

Tabla 4. Donantes habituales por grupo sanguíneo y Factor Rh positivo en ambos géneros, Neiva Huila 2013-2017.

\begin{tabular}{ccccccccccccc}
\hline \multirow{2}{*}{ AÑO } & \multicolumn{3}{c}{ A Positivo } & \multicolumn{3}{c}{ AB Positivo } & \multicolumn{3}{c}{ B Positivo } & \multicolumn{3}{c}{ O Positivo } \\
& No & $\%$ & IC 95\% & No & $\%$ & IC 95\% & No & $\%$ & IC 95\% & No & $\%$ & IC 95\% \\
\hline $\mathbf{2 0 1 3}$ & 35 & 3,7 & $2,6-5,1$ & 0 & 0,00 & $0,0-0,4$ & 14 & 1,5 & $0,8-2,4$ & 100 & 10,6 & $8,7-12,6$ \\
$\mathbf{2 0 1 4}$ & 38 & 4,0 & $2,9-5,4$ & 3 & 0,32 & $0,11-0,93$ & 13 & 1,4 & $0,8-2,3$ & 102 & 10,8 & $8,9-12,9$ \\
$\mathbf{2 0 1 5}$ & 48 & 5,1 & $3,8-6,6$ & 3 & 0,32 & $0,11-0,93$ & 19 & 2,0 & $1,2-3,1$ & 99 & 10,5 & $8,6-12,5$ \\
$\mathbf{2 0 1 6}$ & 59 & 6,2 & $4,8-7,9$ & 4 & 0,42 & $0,16-1,08$ & 9 & 1,0 & $0,5-1,8$ & 142 & 15,0 & $12,8-17,4$ \\
$\mathbf{2 0 1 7}$ & 51 & 5,4 & $4,1-7,0$ & 0 & 0,00 & $0,0-0,4$ & 7 & 0,7 & $0,3-1,5$ & 129 & 13,6 & $11,5-15,9$ \\
TOTAL & 231 & 24,4 & $21,7-27,2$ & 10 & 1,06 & $0,5-1,9$ & 62 & 6,5 & $5,1-8,3$ & 572 & 60,4 & $57,2-63,4$ \\
\hline \hline
\end{tabular}

Fuente: Elaboración propia a partir de la base de datos del banco de sangre.

Los donantes voluntarios de sangre habitual con $\mathrm{Rh}$ negativo se presentaron en menor proporción durante las jornadas de promoción y captación de sangre, representando el 7,2\% (I.C ${ }_{95 \%}: 6,0-9,4$ ) del total de las donaciones habituales, el grupo sanguíneo con mayor frecuencia entre los Rh negativos fue el grupo $\mathrm{O}$ (Ver tabla 5).

Tabla 5. Distribución de los donantes habituales por grupo sanguíneo y Factor Rh Negativo.

\begin{tabular}{ccccccccccccc}
\hline AÑO & \multicolumn{3}{c}{ A Negativo } & \multicolumn{3}{c}{ AB Negativo } & \multicolumn{3}{c}{ B Negativo } & \multicolumn{3}{c}{ O Negativo } \\
& No & $\%$ & IC 95\% & No & $\%$ & IC 95\% & No & $\%$ & IC 95\% & No & $\%$ & IC 95\% \\
\hline $\mathbf{2 0 1 3}$ & 4 & 0,4 & $0,1-1,0$ & 2 & 0,2 & $0,0-0,7$ & 0 & 0,0 & $0,0-0,4$ & 7 & 0,7 & $0,3-1,5$ \\
$\mathbf{2 0 1 4}$ & 3 & 0,3 & $0,1-0,9$ & 1 & 0,1 & $0,0-0,6$ & 0 & 0,0 & $0,0-0,4$ & 5 & 0,5 & $0,2-1,2$ \\
$\mathbf{2 0 1 5}$ & 5 & 0,5 & $0,2-1,2$ & 1 & 0,1 & $0,0-0,6$ & 2 & 0,2 & $0,0-0,7$ & 9 & 1,0 & $0,5-1,8$ \\
$\mathbf{2 0 1 6}$ & 5 & 0,5 & $0,2-1,2$ & 0 & 0,0 & $0,0-0,4$ & 3 & 0,3 & $0,1-0,9$ & 6 & 0,6 & $0,2-1,3$ \\
$\mathbf{2 0 1 7}$ & 4 & 0,4 & $0,1-1,0$ & 0 & 0,0 & $0,0-0,4$ & 4 & 0,4 & $0,1-1,0$ & 11 & 1,2 & $0,6-2,0$ \\
TOTAL & 21 & 2,2 & $1,4-3,3$ & 4 & 0,4 & $0,1-1,0$ & 9 & 1,0 & $0,5-1,8$ & 38 & 4,0 & $0,9-5,4$ \\
\hline
\end{tabular}

Fuente: Elaboración propia a partir de la base de datos del banco de sangre.

\section{Discusión}

La sangre y los componentes anatómicos se obtienen gracias a la donación altruista, gratuita y desinteresada de los seres humanos, quie- nes se han convertido en salvavidas, en aras de mejorar la calidad de vida de algunos pacientes crónicos(10) o en condiciones vulnerables de salud. La decisión de ser donante o no, puede llegar a ser influenciada por factores demográficos como el género y la edad; por tal razón, los 
promotores de esta práctica deben conocer y definir la población a intervenir para así, crear estrategias efectivas que permitan la fidelización de los donantes(11).

Mediante este estudio se observó que en el año 2016 se presentó un mayor número de donaciones, lo cual coincide con lo presentado por el Ministerio de Salud en las estadísticas anuales. Teniendo en cuenta que para el año 2017 se captaron 830.291 unidades de sangre total a partir de 1'003.314 donantes potenciales, esto representa un incremento en la captación del 1,6\% con respecto a 2016 a pesar de que el número potencial de donantes se redujo en $0,1 \%(9)$.

En el mismo sentido, según el último informe presentado por el Ministerio de Salud se evidenció que la donación voluntaria repetida ha presentado un incremento, pasando del 21\% al 22,1\% (9). En el estudio realizado en Corea del Sur por Lee et al.(12), se publicó que los donantes de sangre ocasionales representaron el $74,8 \%$ y los habituales el $25,2 \%$; contrario a los resultados registrados por Sánchez et al.(13) en Cuba, quienes hallaron una distribución de donantes ocasionales del $26,1 \%$ y de habituales del $73,8 \%$, porcentajes similares obtenidos por el presente estudio en Neiva. Estos datos resaltan la importancia de implementar nuevas estrategias de promoción de donación de sangre con el fin de incrementar la habitualidad en los donantes, ya que, de acuerdo con Charbonneau et al.(14), existen perfiles de donantes específicos al compararse la demografía y las motivaciones de las donaciones, por lo que concluye que las agencias deben desarrollar nuevas estrategias de reclutamiento adaptadas a estos donantes, especialmente si se desea convencerlos para que se conviertan en donantes de aféresis o habituales.

Entre la caracterización demográfica de los donantes voluntarios de sangre durante el periodo 2013-2017, se observa que el género más representativo es el masculino. Según la OMS, de los 118 países que aportan información respecto al género de los donantes de sangre, se sabe que el $30 \%$ de las donaciones provienen de mujeres y que existen 18 países en donde la donación femenina representa menos del 10\%(2). En Colombia, el 47,4\% de las donaciones provienen de mujeres(9), datos similares a los registrados en Brasil por Gonçalez et al.(15), en cuya investigación el género femenino representó el 34\% de la población. Por el contrario, en Tanzania según Jahanpor et al.(16), las mujeres representaron el $12 \%$ de la población donante.

La tendencia en el género de los donantes se ex- plica en que los hombres presentan una fisiología diferente a las mujeres, son menos propensos a ser diferidos temporalmente por razones médicas como: niveles bajos de hemoglobina, bajo peso corporal $(<50 \mathrm{~kg})$ y experimentar eventos adversos durante la donación(17). En Colombia, según el Instituto Nacional de Salud, los hombres pueden donar cada tres meses y las mujeres cada cuatro meses (18). Datos similares se evidenciaron en la India entre el periodo 2012 - 2015, en donde la desigualdad entre hombres y mujeres hace que este último presente un porcentaje bajo en las donaciones(19). Otro factor que puede incidir en la frecuencia de las donaciones en las mujeres es el biológico, dado por el ciclo menstrual, embarazo y lactancia materna, aunque también se resaltan factores como psicológicos, sociales y culturales(20).

En términos de edad, la población en Colombia puede donar sangre a partir de los 18 años hasta los 65 años(3). Al observar por rangos de edad, se evidencia que la población donante en su mayoría es la más joven, datos que coinciden con lo reportado anualmente por el Instituto Nacional de Salud (2013-2017), que establece que las edades con mayor número de donaciones se encuentran entre los 18 y 30 años(9,21-23).

En diversos estudios realizados a nivel global se evidenció esta tendencia, como es el caso de Nigeria durante el periodo 2016-2017 por Ugwu et al. (24), en donde los jóvenes entre 18 y 27 años presentaron un mayor porcentaje de donaciones en el 55\% de los casos. En investigación publicada en el 2017 en Dinamarca, se encontró que las mujeres mayores de 25 años mantuvieron una prevalencia del $7,5 \%$, la cual se disminuía proporcionalmente a medida que aumentaba la edad, los autores concluyen que este fenómeno podría estar dado a la maternidad y a la lactancia, mientras que en los hombres la prevalencia de las donaciones se mantuvo 5,5\%, en edades entre 25 y 55 años(25). Para Alemania las tasas de donación de sangre por 1.000 habitantes aumentaron durante 2005 y 2010 en los jóvenes y luego disminuyeron en 2015. La distribución de edad de los donantes ya no es estática, sino que cambia constantemente hacia grupos de mayor edad, en paralelo con el envejecimiento de la población, lo que provoca una mayor reducción en las donaciones de sangre en los grupos de edad de 50 a 60 años, que no podrán donar sangre por razones de salud; se concluyó que la disminución en las donaciones de sangre es paralela al cambio demográfico (26).

En Japón, un país con cambios demográficos 
constantes, un estudio publicado en el 2016 por Akita et al.,(27), reportó un incremento en las donaciones de sangre en personas entre 20 y 30 años, que fue disminuyendo cada año; el estudio concluyó que si los comportamientos actuales de donación de sangre continúan, es probable que ocurra un déficit de disponibilidad de sangre en Japón. Así mismo, las donaciones de sangre insuficientes se relacionaron principalmente con una reducción proyectada en la población de 20 a 30 años, por lo que es crucial reclutar y retener nuevos donantes (27). En resumen, los datos de los estudios hechos en Dinamarca, Nigeria, Alemania y Japón, sumados a los encontrados en la presente investigación, proveen información útil a los gobiernos para que garanticen la adecuación del suministro de sangre mediante la creación de estrategias de promoción de donaciones adecuadas para el tipo de población.

La otra variable revisada, el factor $\mathrm{Rh}$, presentó una mayor frecuencia en el Positivo con respecto al Negativo dentro de los donantes voluntarios de sangre. Frente al grupo sanguíneo, el grupo $\mathrm{O}$ predomina en esta población de estudio, datos que coinciden con los estudios realizados en Córdoba (Colombia) por Causil et al. (28) y en Antioquia (Colombia) por Flórez et al. (29), en los cuales el $\mathrm{Rh}$ positivo tuvo la mayor prevalencia, al igual que el grupo sanguíneo O. Esta tendencia es similar a los encontrado en Venezuela por Vizcaya et al. (30).

En otros países, la tendencia es variada, en África son comunes los resultados en los que el grupo sanguíneo predominante entre los donantes fue $\mathrm{O}$ y el menos predominante fue $\mathrm{AB}$, como se evidencia en estudios realizados en Ghana(31-32) y Uganda(33). En algunos países de Asia, el grupo sanguíneo que predomina en los donantes es el grupo $\mathrm{O}$, seguido por el grupo A y luego el grupo B como lo evidencia en Japón Tsuchimine et al. (34). Muy diferente son las frecuencias de los estudios realizado en la India por Singh et al.(19) y por Garg et al.(35), en donde el grupo sanguíneo común fue $\mathrm{B}$ y el menos común $\mathrm{AB}$. Por su parte, en un estudio realizado en Alemania por Chandler et al.(36), se presenta que el grupo sanguíneo con más prevalencia es el A y el Rh con más prevalencia es el positivo. Al evidenciar las diferentes frecuencias en distintas partes del mundo, es posible evidenciar la distribución demográfica de los grupos sanguíneos y notar que el grupo sanguíneo AB es el menos distribuido entre la población del mundo, datos que coinciden con el presente estudio.

\section{Conclusiones}

- Al analizar las características género y edad de los donantes voluntarios habituales de sangre por año, se observó que el 87,5\% de las donaciones habituales se encuentran concentradas en el rango de edad, entre los 16 y 29 años para ambos géneros, pero simultáneamente presenta una disminución cercana al $90 \%$ en los donantes habituales después de los 30 años. Así mismo, se encontró que los hombres tuvieron una mayor participación en las jornadas de promoción y captación de sangre durante el periodo estudiado, dada por la hipótesis que los hombres pueden realizar un mayor número de donaciones en el año en comparación con las mujeres. La segunda hipótesis que surge de esta investigación es que a medida que aumentan las donaciones de sangre habitual en los grupos de edad comprendido entre los 16-29, estas disminuyen en igual proporción en los grupos de edades mayores a los 30 años, lo cual podría estar relacionado a que la población a esta edad es menor en las instituciones universitarias.

- Los resultados han demostrado que la estrategia implementada por el Banco de Sangre en la promoción y captación de sangre en la unidad móvil en las instituciones universitarias ha sido de gran ayuda para mantener un inventario de hemoderivados, lo cual le ha permitido salvar miles de vidas en la población del Huila; sin embargo, continúa siendo insuficiente para cubrir las necesidades de la región.

- Se recomienda fortalecer las investigaciones al interior de las instituciones de educación superior en las experiencias vividas por los donantes de sangre posterior al proceso de extracción de sangre y el fenómeno de la reducción de frecuencia en las donaciones por parte de los donantes, posterior a la tercera década de la vida. Los promotores de esta práctica deben conocer y definir la población a intervenir, para así crear estrategias efectivas que permitan la fidelización de los donantes.

Declaración de conflictos de intereses: Los autores expresamos abiertamente que no existieron conflictos de intereses para el desarrollo de la presente investigación y declaramos que la Universidad Surcolombiana fue la única fuente de financiación, mediante convocatoria interna, para conformar el banco de proyectos de semilleros de investigación y desarrollo tecnológico e innovación, en la modalidad de financiación.

Rev. cienc. cuidad. 2020;17(3):75-84 
Agradecimientos: Los autores agradecen el apoyo recibido por la Unidad de estadística de la Facultad de Salud de la Universidad Surcolombiana. Igualmente expresan su agradecimiento al Banco de Sangre de la E.S.E Hospital Universitario Hernando Moncaleano Perdomo de la ciudad de Neiva-Huila y a la Universidad Surcolombiana por el apoyo económico para realizar esta investigación, finalmente a docentes, estudiantes y egresados que hacen parte del proyecto "Promoción de la donación voluntaria y habitual de sangre en la comunidad huilense".

\section{Referencias bibliográficas}

1. Organización Mundial de la Salud. Sangre y componentes seguros: estableciendo un programa de aprendizaje a distancia en seguridad sanguínea: una guía para coordinadores de programa. Ginebra: Organización Mundial de la Salud; 1998. 10-13 p.

2. Organización Mundial de la Salud. Global Status Report on Blood Safety and Availability. Ginebra; 2016.

3. Ministerio de Salud y Protección Social. El desafío es lograr incrementar la donación de sangre en Colombia. 2018.

4. Zucoloto ML, Gonçalez TT, Gilchrist PT, Custer B, McFarland W, Martinez EZ. Factors that contribute to blood donation behavior among primary healthcare users: A structural approach. Transfus Apher Sci. 2019 Oct;58(5):663-8.

5. Instituto Nacional de Salud, Ministerio de Salud, Lineamiento técnico para la selección de donantes de sangre en Colombia; 2018. 87-89 p.

6. Organización Mundial de la Salud. El uso clínico de la sangre. 2001.

7. Organización Panamericana de la Salud, Organización Mundial de la Salud, $48^{\circ}$ Consejo Directivo. Resolución CD48.57. Washington; 2008.

8. Ministerio de Salud. Decreto 1571 de 1993. Bogotá D.C.; 1993.

9. Instituto Nacional de Salud, Red Nacional de Bancos de Sangre y Servicios de Transfusión. Informe anual Red Nacional de Bancos de Sangre y Servicios de Transfusión, Colombia 2017. 2018.

10. Organización Panamericana de la Salud, Organización Mundial de la Salud. Estrategia para el acceso universal a la salud y la cobertura universal de salud. Washington; 2014.

11. Olivera Cuadra D, Cárdenas Carvajal M, Ferrera Morales B. La promoción de donación de sangre ante la necesidad de la obtención de un producto seguro. Medicentro Electrónica. 2019;23(2):1259.

12. Lee H-J, Shin K-H, Song D, Lee S-M, Kim I-S, Chang CL, et al. Analysis of Blood Donors in a Tertiary Care Hospital as a Fixed Collection Site. Korean J Blood Transfus. 2017 Apr;28(1):28-35.

13. Sánchez Frenes P, Hernández Malpica S, Rojo Pérez N, Bayarre Vea HD, Pérez Ulloa LE. Implicaciones de los cambios demográficos en la disponibilidad de sangre del sistema sanitario de Cienfuegos. Rev Cuba Salud Pública. 2017;43(4).

14. Charbonneau J, Cloutier M-S, Carrier É. Whole blood and apheresis donors in Quebec, Canada: Demographic differences and motivations to donate. Transfus Apher Sci. 2015 Dec;53(3):320-8.

15. Gonçalez TT, Sabino EC, Schlumpf KS, Wright DJ, Mendrone A, Lopes M, et al. Analysis of donor deferral at three blood centers in Brazil. Transfusion. 2013 Mar;53(3):531-8.

16. Jahanpour O, Pyuza JJ, Ntiyakunze EO, Mremi A, Shao ER. ABO and Rhesus blood group distribution and frequency among blood donors at Kilimanjaro Christian Medical Center, Moshi, Tanzania. BMC Res Notes. 2017 Dec;10(1):738.

17. Carver A, Chell K, Davison TE, Masser BM. What motivates men to donate blood? A systematic review of the evidence. Vox Sang. 2018 Apr;113(3):205-19.

18. Instituto Nacional de Salud. ¿Quieres ser donante de sangre? Donación de Sangre.

19. Singh A, Srivastava R, Deogharia K, Singh K. Distribution of ABO and Rh types in voluntary Blood donors in Jharkhand area as a study conducted by RIMS, Ranchi. J Fam Med Prim Care. 
2016;5(3):631.

ISSN-PRINT

1794-9831

E-ISSN 2322-7028

Vol. 17 No. 3

Sep - Dic 2020

Cúcuta, Colombia

20. Singh DS. Pattern and Trend of Female Donors in Blood Bank and Outdoor Blood Donation Camps - A 7 year Comparative Study from Blood Bank, Chamba, (H.P). J Med Sci Clin Res. 2018 Jul;6(7).

21. Red Nacional de Bancos de Sangre y Servicios de Transfusión, Instituto Nacional de Salud. Informe Nacional Indicadores 2013. 2013.

22. Instituto Nacional de Salud, Red Nacional de Bancos de Sangre y Servicios de Transfusión. Informa Anual Red de Sangre 2015. 2016.

23. Instituto Nacional de Salud, Red Nacional de Bancos de Sangre y Servicios de Transfusión. Informe Anual Red de Sangre 2014. 2015.

24. Ugwu A, Efobi C, Ibegbulam O, Madu A. Pattern of blood donation and characteristics of blood donors in Enugu, Southeast Nigeria. Niger J Clin Pract. 2018;21(11):1438-43.

25. Burgdorf KS, Simonsen J, Sundby A, Rostgaard K, Pedersen OB, Sørensen E, et al. Socio-demographic characteristics of Danish blood donors. Bönig H, editor. PLoS One. 2017 Feb;12(2).

26. Greinacher A, Weitmann K, Schönborn L, Alpen U, Gloger D, Stangenberg W, et al. A population-based longitudinal study on the implication of demographic changes on blood donation and transfusion demand. Blood Adv. 2017 Jun;1(14):867-74.

27. Akita T, Tanaka J, Ohisa M, Sugiyama A, Nishida K, Inoue S, et al. Predicting future blood supply and demand in Japan with a Markov model: application to the sex- and age-specific probability of blood donation. Transfusion. 2016 Nov;56(11):2750-9.

28. Causil L, Gómez L, Otero D, Moscote L, Monterrosa L, Burgos D et al. Frecuencia de grupos sanguíneos (sistema ABO) entre estudiantes de la Universidad de Córdoba, sede Berástegui. CIMEL 2016;21(1):24-27.

29. Flórez-Duque J, Gómez-Álvarez A, Patiño Carreño J, Cardona-Arias JA. Prevalencia de anticuerpos irregulares en donantes en un banco de sangre de Antioquia, 2016-2018. Rev CES Med 2019; 33(1): 3-12.

30. Vizcaya, T., Colmenares, M., Pérez, L., Díaz, A., Pineda, A., \& Duarte, Y. (2019). Distribución de grupos sanguíneos ABO y $\mathrm{Rh}$ en candidatos a donantes de el Tocuyo, Venezuela. Revista Venezolana De Salud Pública, 7(2), 9-16.

31. Doku GN, Agbozo WK, Annor RA, Kisseh GD, Owusu MA. Frequency of ABO/Rhesus (D) blood groupings and ethnic distribution in the Greater-Accra region of Ghana, towards effective blood bank inventory. Int J Immunogenet. 2019 Apr;46(2):67-73.

32. Nkansah C, Serwaa D, Osei-Boakye F, Owusu-Ampomah R. Seroprevalence and trend of hepatitides among blood donors in a district hospital in Ghana: a nine-year retrospective, descriptive cross-sectional study. J Immunoass Immunochem. 2020 Jan;41(1):71-83.

33. Apecu RO, Mulogo EM, Bagenda F, Byamungu A. ABO and Rhesus (D) blood group distribution among blood donors in rural south western Uganda: a retrospective study. BMC Res Notes. 2016 Dec;9(1).

34. Tsuchimine S, Saruwatari J, Kaneda A, Yasui-Furukori N (2015) ABO Blood Type and Personality Traits in Healthy Japanese Subjects. PLoS ONE 10(5): e0126983. https://doi.org/10.1371/ journal.pone. 0126983

35. Garg P. Prevalance of ABO and Rhesus Blood Groups in Blood Donors: A Study from a Tertiary Care Teaching Hospital of Kumaon Region of Uttarakhand. J Clin Diagnostic Res. 2014;8(12):FC16-9.

36. Chandler T, Hiller J, Peine S, Stargardt T. Blood donation and donors: insights from a large German teaching hospital (2008-2017). Vox Sang. 2020;115(1):27-35. https://oi.org/doi:10.1111/ vox. 12853 